diminished central motor output required to drive the respiratory muscles. Thus, the associated perceived effort required to generate a given ventilation during exercise is similarly reduced. The deeper, slower breathing pattern after radiation therapy and delay in the onset of tachypnoea reflect the recruitment of IC both at rest and during exercise as a result of lung volume deflation. Thus, the patient could increase tidal volume and ventilation during exercise to a greater extent than previously, before reaching critical mechanical constraints. For this reason, the onset of intolerable dyspnoea was delayed and the limits of tolerance extended.

Ventilatory requirements also decreased after radiation, presumably reflecting a net improvement in the ventilationperfusion relationship. Thus, measurements of ventilatory inefficiency improved, reflecting more effective carbon dioxide elimination and reduced wasted ventilation. Arterial oxygen saturation was unaffected throughout exercise. The $27 \%$ reduction in $D \mathrm{~L}, \mathrm{CO}$ is a well-documented effect of radiation injury to the pulmonary vasculature and indicates a reduction in the surface area for pulmonary gas exchange.

The clinical decision to offer radical radiotherapy to individuals with severe COPD with early-stage lung carcinoma is often difficult given the known negative consequences of further erosion of an already limited ventilatory reserve. Given the vast pathophysiological heterogeneity of COPD, the impact of targeted radiotherapy is likely to be highly variable. It is conceivable, as our case illustrates, that in some patients with extensive lung hyperinflation and emphysema, radiationinduced alterations in the elastic properties of the lung and in ventilation-perfusion mismatching may actually have favourable effects on dyspnoea and exercise tolerance. Prospective studies to comprehensively characterise COPD phenotypes and measure the effects of radiation on respiratory physiology and patient-centred outcomes are required to better refine selection criteria for radiotherapy in this population.

P. O'Meara*, J.A. Guenette*, N. Raghavan*, N. Amornputtisathaporn*, C.E. deMetz", R.L. Nolan" and D.E. O'Donnell*

*Respiratory Investigation Unit, Dept of Medicine, Queen's University and Kingston General Hospital, "Cancer Centre of
South Eastern Ontario, Kingston General Hospital, and "Dept of Diagnostic Radiology, Kingston General Hospital, Kingston, ON, Canada.

Correspondence: D.E. O'Donnell, Division of Respiratory and Critical Care Medicine, Dept of Medicine, Queen's University, 102 Stuart Street, Kingston, Ontario, K7L-2V6, Canada. E-mail: odonnell@queensu.ca

Support Statement: J.A. Guenette was supported by the John Alexander Stewart Fellowship (Dept of Medicine, Queen's University and Kingston General Hospital, Kingston, ON, Canada) and the Natural Sciences and Engineering Research Council of Canada Post Doctoral Fellowship. N. Raghavan was supported by the John Alexander Stewart Fellowship.

Statement of Interest: A statement of interest for D.E. O'Donnell can be found at www.erj.ersjournals.com/site/ misc/statements.xhtml

\section{REFERENCES}

1 Ghafoori P, Marks LB, Vujaskovic Z, et al. Radiation-induced lung injury. Assessment, management, and prevention. Oncology (Williston Park) 2008; 22: 37-47.

2 Axford AT, Cotes JE, Deeley TJ, et al. Clinical improvement of patients with emphysema after radiotherapy. Thorax 1977; 32: 35-39.

3 Abratt RP, Morgan GW. Lung toxicity following chest irradiation in patients with lung cancer. Lung Cancer 2002; 35: 103-109.

4 Hoffbrand BI, Gillam PM, Heaf PJ. Effect of chronic bronchitis on changes in pulmonary function caused by irradiation of the lungs. Thorax 1965; 20: 303-308.

5 Boushy SF, Helgason AH, North LB. The effect of radiation on the lung and bronchial tree. Am J Roentgenol Radium Ther Nucl Med 1970; 108: 284-292.

6 Laghi F, Jubran A, Topeli A, Fahey PJ, et al. Effect of lung volume reduction surgery on neuromechanical coupling of the diaphragm. Am J Respir Crit Care Med 1998; 157: 475-483.

7 O'Donnell DE, Hamilton AL, Webb KA. Sensory-mechanical relationships during high-intensity, constant-work-rate exercise in COPD. J Appl Physiol 2006; 101: 1025-1035.

DOI: $10.1183 / 09031936.00029411$

\title{
Linezolid safety, tolerability and efficacy to treat multidrug- and extensively drug-resistant tuberculosis
}

\section{To the Editors:}

Treatment of multidrug-resistant (MDR) tuberculosis (TB) (defined as in vitro resistance to at least isoniazid and rifampicin, the two most potent first-line drugs for TB treatment) and extensively drug-resistant (XDR)-TB (defined as in vitro resistance to isoniazid and rifampicin plus any fluoroquinolone and at least one of the injectable drugs: amikacin, capreomycin or kanamycin) is still a major problem from both a clinical and a public health perspective [1-5].

Treatment outcomes for complicated MDR-TB cases (those with additional resistance beyond isoniazid and rifampicin) and XDR-TB cases being still poor, the need for information on the safety, tolerability and efficacy of other antibiotics that are potentially useful in their treatment is urgent [6-9]. 


\begin{tabular}{ll}
\hline TABLE 1 & $\begin{array}{l}\text { Sociodemographic and clinical characteristics of } \\
16 \text { multidrug-/extensively drug-resistant } \\
\text { tuberculosis patients exposed to linezolid }\end{array}$ \\
XDR-TB & $12 / 16(75)$ \\
Drug resistance & \\
Streptomycin & $15 / 16(93.8)$ \\
Ethambutol & $13 / 16(81.3)$ \\
Pyrazinamide & $15 / 16(93.8)$ \\
Fluoroquinolones & $16 / 16(100)$ \\
Amikacin & $7 / 16(43.8)$ \\
Kanamycin & $9 / 11(81.8)$ \\
Capreomycin & $16 / 16(100)$ \\
Previous exposure to anti-TB therapy for & $9 / 16(56.3)$ \\
$\quad>\mathbf{2 0}$ days & \\
Number of times treated with anti-TB & $1.5(0-3)$ \\
$\quad$ drugs for $>\mathbf{1}$ month & $14 / 16(87.5)$ \\
Sputum smear converters & $14 / 16(87.5)$ \\
Culture converters & $82(34-152)$ \\
Duration of hospital stay days &
\end{tabular}

Data are presented as $\mathrm{n} / \mathrm{N}$ (\%) or median (interquartile range). XDR-TB: extensively drug-resistant tuberculosis (TB)

In vitro and pharmacological data suggest that linezolid, an oxazolidinone antibiotic, could be useful in treating mycobacterial infections, including MDR-TB [9-11]. However, clinical experience with the off-label use of linezolid is still limited to case reports and small case series involving nontuberculous mycobacterial diseases [12] and TB, the four largest cohorts including 10 [13], 12 [14], 30 [15] and 85 cases (but only 45 with information on efficacy) [9], respectively. The aim of this study was to evaluate the safety, tolerability and efficacy of linezolid in a cohort of patients with MDR/XDR-TB from Portugal.

Clinical information necessary to study safety, tolerability and efficacy was prospectively collected on all MDR-TB cases treated with linezolid in Portugal between 2004 and 2009, with the exception of one case who started the treatment in 2003 (whose information was collected retrospectively). The protocol was designed to be compatible with that used to study the largest cohort available [9]. Internationally agreed outcome definitions were used [9].

In particular, a patient who completed treatment and was consistently culture-negative with at least five results for the final 12 months of treatment was defined as cured. If bacteriological results were lacking (i.e. $<5$ cultures performed), the case was defined as treatment completed. Treatment failure was defined as two or more positive cultures in the previous 12 months of treatment, or if a medical decision was made to terminate treatment due to poor response or adverse events. Definitions of other treatment outcomes (i.e. death, default and transfer) were the same as previously used in the European study on linezolid efficacy, safety and tolerability [9].

Approval for collection of study data was provided by the ethical committee of the coordinating centre [9], the participating hospitals complying with national regulations and organisational requirements for protection of human subjects. All data were coded and individual identifiers were available only to treating physicians.
Safety and tolerability end-points included major and minor side-effects. A major side-effect was defined as any adverse reaction that resulted in temporary or permanent discontinuation of linezolid, while a minor side-effect required only dose adjustment and/or addition of concomitant treatment.

Efficacy end-points included time to and proportion of sputum smear and culture conversions, and treatment outcome. Sputum conversion was defined as two consecutive negative sputum smears in patients who were sputum smear-positive at diagnosis. Time to culture conversion was defined as time from treatment start to date of the first of two consecutive negative cultures.

Drug susceptibility testing (DST) for all first- and second-line anti-TB drugs was performed by the Supranational Reference Laboratory in Porto, Portugal (quality-assured within the World Health Organization (WHO) proficiency scheme) [1, 2]. All participating centres performed sputum smear examinations weekly until negative, and then monthly. Cultures were performed monthly.

Regimens to treat MDR/XDR-TB cases were tailored to DST results according to $\mathrm{WHO}$ recommendations, using fluoroquinolones, injectable agents and other second-line oral agents coadministered with linezolid in all individuals. Linezolid was available without any limitation, and was prescribed at the dose of 1,200 mg once daily to 15 cases except one, who received $600 \mathrm{mg}$ once daily.

The study end-points have been compared with those of the two largest series, including only patients with a definitive treatment outcome in the efficacy analyses (cure, failure and death) $[9,15]$.

Categorical variables were compared using the Chi-squared test and continuous variables by the t-test, or Wilcoxon-MannWhitney for unpaired data. A p-value of $\leqslant 0.05$ was considered statistically significant. Data were collected on standardised eforms and analysed using Stata 9.0 (StataCorp, College Station, TX, USA).

The main results are summarised in table 1.16 cases were analysed, $12(75 \%)$ being affected by XDR-TB strains. $12(75 \%)$ were males, with a mean \pm SD age of $34.8 \pm 10$ yrs. Five $(31.3 \%)$ were foreign-born immigrants (three from Cape Verde, one from Angola and one from Guinea-Bissau, respectively). Six $(37.5 \%)$ were HIV seropositive, all of them being treated with anti-retroviral drugs. $12(75 \%)$ of them were affected by pulmonary and four had simultaneous extrapulmonary forms (three pleural and one lymph node TB, respectively) of culturepositive disease. Three $(18.8 \%)$ cases underwent surgical interventions (pleurectomy, lobectomy and pneumonectomy, respectively). The median duration of hospital stay in the cohort was 82 days (interquartile range (IQR) 34-152 days).

Out of 16 cases, nine completed their treatment (eight cured, one died), one defaulted at month 20 after achieving sputum smear and culture conversion, and six were still on treatment at the time of the analysis (four out of six having converted both sputum smear and culture after an average time of 275 and 650 days, respectively).

Linezolid was administered for a median (IQR) period of 375 days (270-630 days). 


\begin{tabular}{|c|c|c|c|c|c|}
\hline & Current study & MIgLIORI et al. [9] & p-value $(95 \% \mathrm{Cl})^{\#}$ & SCHECTER et al. [15] & p-value $(95 \% \mathrm{Cl})^{*}$ \\
\hline \multicolumn{6}{|l|}{ Treatment outcome } \\
\hline Success & 8/9 (88.9) & $36 / 45(80.0)$ & $0.53(-15-33)$ & 22/23 (95.7) & $0.45(-29-15)$ \\
\hline Cured & $8 / 9(89)$ & & & & \\
\hline Failure & $0 / 9(0)$ & $0 / 45(0)$ & & $1 / 23(4.3)$ & \\
\hline Death & $1 / 9(11.1)$ & $9 / 45(20.0)$ & $0.53(-33-15)$ & $0 / 23(0)$ & - \\
\hline Default & $1 / 16(6.3)$ & & & & \\
\hline Still on treatment & 6/16 (38) & & & & \\
\hline Time to sputum smear conversion days & $150(60-540)$ & $76(56-162)$ & 0.07 & & \\
\hline Time to culture conversion days & $180(90-1380)$ & $108(56-160)$ & 0.02 & & \\
\hline
\end{tabular}

Three $(18.8 \%)$ cases experienced adverse events, including anaemia, pancytopenia and polyneuropathy, after 30, 63 and 60 days of drug exposure, respectively.

Comparing the main outcomes with those of the main series (table 2), we observed no difference in successful treatment outcomes $(89 \%$ in this study versus $80 \%$ in the European cohort [9] and $96 \%$ in the cohort of SCHECTER et al. [15], p-value not significant).
The median time to sputum smear and culture conversion was shorter in the European study than in the present study (76 versus 150 days, $p=0.07$; and 108 versus 180 days, $p=0.02$, respectively).

The proportion of major adverse events was significantly lower in Portugal than in the European cohort [9] (one out of 16 versus 27 out of $85 ; \mathrm{p}=0.03$ ) but not significantly different if compared with that recorded in the USA (one out of 16 versus three out of 30; $\mathrm{p}=0.65$ ) [15].

\section{TABLE 3 Anti-tuberculosis regimens prescribed to multidrug (MDR)-/extensively drug-resistant tuberculosis (TB) patients in Portugal, 2003-2009}

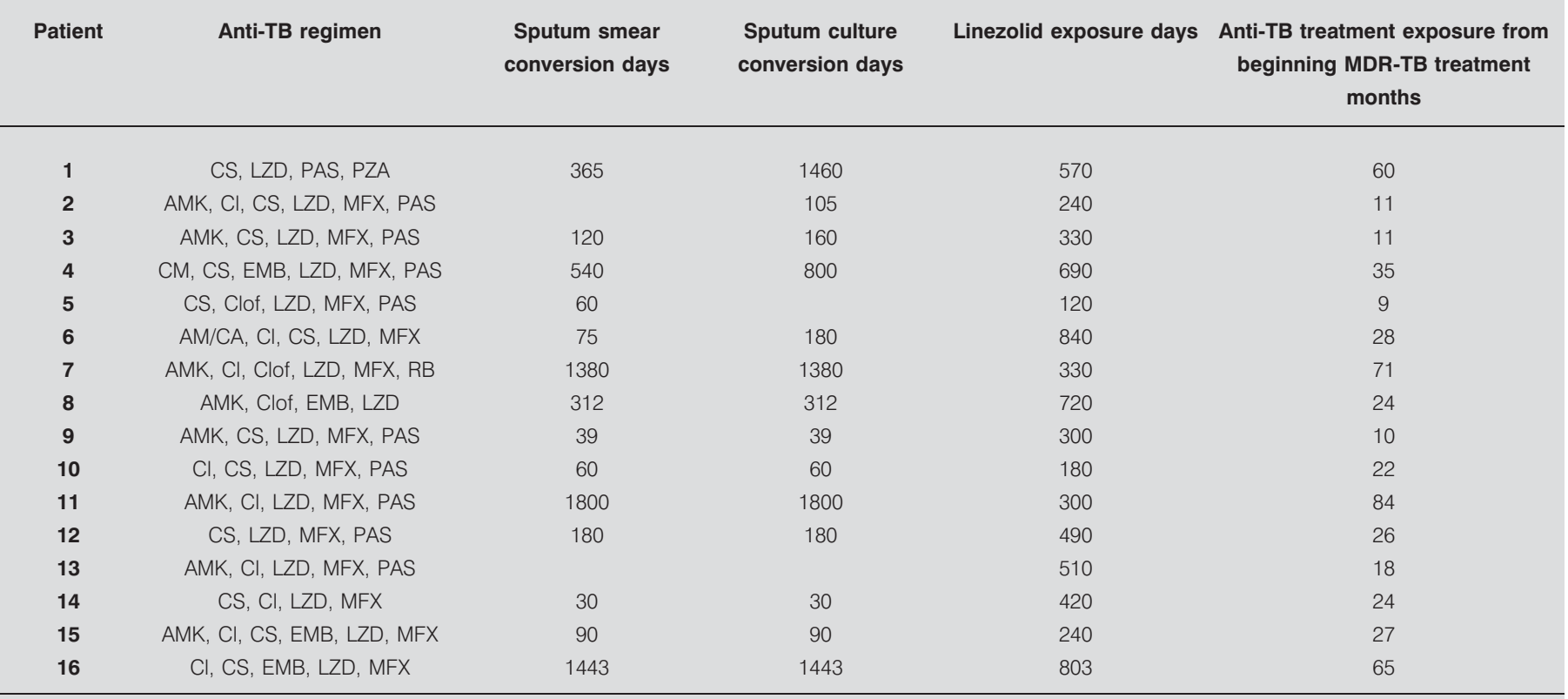

CS: cycloserine; LZD: linezolid; PAS: p-aminosalicylic acid; PZA: pyrazinamide; AMK: amikacin; Cl: clarithromycin; MFX: moxifloxacin; CM: capreomycin; EMB: ethambutol; Clof: clofazimine; AM/CA: amoxicillin/clavulanic acid; RB: rifabutin. 
No significant difference was found when the comparison for minor adverse events was performed.

The regimens used to treat individual patients and information on bacteriological conversion are summarised in table 3 .

The results of this observational study confirm that under specialised management, treatment success in difficult-to treat MDR-/XDR-TB cases is high, although the proportion of XDRTB cases (and of HIV-positive) individuals in Portugal is higher than in the other major studies available $[9,15]$. Furthermore, a control group of MDR-/XDR-TB patients treated with linezolid-sparing regimens was not analysed. However, the proportion of adverse events (both minor and major) was lower than in previous cohorts, being significant only when comparing major adverse events of the Portuguese cohort versus a European one [9], although a relatively high dose of linezolid (1,200 $\mathrm{mg}$ once daily) was used.

The evaluation of safety and tolerability is difficult to perform, as different drugs are included in the treatment regimens (necessarily guided by DST) and the duration of exposure to linezolid, as well as the dosages prescribed (300-1,200 mg), varied among patients and among studies.

Pending larger studies, a meta-analysis including individual data from the patients treated with linezolid will be important to hopefully give the final word on whether this drug could really be considered for wider use outside the few cases affected by strains resistant to more than seven drugs [9].

\section{Villar*\#++, G. Sotgiu ${ }^{\text {I++ }}$, L. D'Ambrosio ${ }^{+}$, E. Raymundo ${ }^{\S}$, L. Fernandes ${ }^{f}$, J. Barbedo**, N. Diogo ${ }^{\# \#}$, C. Lange ${ }^{\uparrow \uparrow}$, R. Centis ${ }^{+}$and G.B. Migliori ${ }^{+}$}

*General Directorate of Health, Lisbon, "Lung Diseases Centre of Venda Nova, Amadora, ${ }^{\S}$ Lung Diseases Centre of Vila Franca de Xira, Vila Franca de Xira, ${ }^{f}$ Lung Diseases Centre of Lumiar, Lisbon, ${ }^{* *}$ Lung Diseases Centre of Sintra, Sintra, and ${ }^{\# \#}$ Pulido Valente Hospital, Lisbon, Portugal. "Hygiene and Preventive Medicine Institute, University of Sassari, Sassari, and, ${ }^{+} \mathrm{WHO}$ Collaborating Centre for TB and Lung Diseases, Fondazione S. Maugeri, Care and Research Institute, Tradate, Italy. " Division of Clinical Infectious Diseases, Research Center Borstel, Borstel, Germany. "+Both authors contributedequally to the study.

Correspondence: G.B. Migliori, World Health Organization Collaborating Centre for Tuberculosis and Lung Diseases, Fondazione S. Maugeri, Care and Research Institute, Via Roncaccio 16, 21049, Tradate, Italy. E-mail: giovannibattista. migliori@fsm.it

Support Statement: The study was supported by the current research funds of the participating institutions. For this publication, the research leading to these results has received funding from the European Community's Seventh Framework Programme (FP7/2007-2013) under grant agreement FP7223681.

Statement of Interest: None declared.

\section{REFERENCES}

1 World Health Organization. Multidrug and extensively drugresistant TB (M/XDR-TB): 2010 global report on surveillance and response. Publication No. WHO/HTM/TB/2010.3. Geneva, World Health Organization, 2010.

2 World Health Organization. Global tuberculosis control 2010. Publication No. WHO/HTM/TB/2010.7. Geneva, World Health Organization, 2010.

3 World Health Organization. Extensively drug-resistant tuberculosis (XDR-TB): recommendations for prevention and control. Wkly Epidemiol Rec 2006; 81: 430-432.

4 Centers for Disease Control and Prevention. Emergence of Mycobacterium tuberculosis with extensive resistance to second-line drugs - worldwide, 2000-2004. MMWR Morb Mortal Wkly Rep 2006; 55: 301-305.

5 Migliori GB, Besozzi G, Girardi E, et al. Clinical and operational value of the extensively drug-resistant tuberculosis definition. Eur Respir J 2007; 30: 623-626.

6 Migliori GB, Lange C, Girardi E, et al. Fluoroquinolones: are they essential to treat multidrug-resistant tuberculosis? Eur Respir J 2008; 31: 904-910.

7 Migliori GB, Lange C, Centis R, et al. Resistance to second-line injectables and treatment outcomes in MDR and XDR-TB cases. Eur Respir J 2008; 31: 1155-1159.

8 Sotgiu G, Ferrara G, Matteelli A, et al. Epidemiology and clinical management of XDR-TB: a systematic review by TBNET. Eur Respir J 2009; 33: 871-881.

9 Migliori GB, Eker B, Richardson MD, et al. A retrospective TBNET assessment of linezolid safety, tolerability and efficacy in multidrug-resistant tuberculosis. Eur Respir J 2009; 34: 387-393.

10 Diekema DJ, Jones RN. Oxazolidinone antibiotics. Lancet 2001; 358: 1975-1982.

11 Molicotti P, Ortu S, Bua A, et al. In vitro efficacy of Linezolid on clinical strains of Mycobacterium tuberculosis and other mycobacteria. New Microbiol 2006; 29: 275-280.

12 Ntziora F, Falagas ME. Linezolid for the treatment of patients with atypical mycobacterial infection: a systematic review. Int J Tuberc Lung Dis 2007; 11: 606-611.

13 Condos R, Hadgiangelits N, Leibert E, et al. Case series report of a linezolid-containing regimen for extensively drug-resistant tuberculosis. Chest 2008; 134: 187-192.

14 Von der Lippe B, Sandven P, Brubakk O. Efficacy and safety of linezolid in multidrug resistant tuberculosis (MDR-TB) - a report of ten cases. J Infect 2006; 52: 92-96.

15 Schecter GF, Scott C, True L, et al. Linezolid in the treatment of multidrug-resistant tuberculosis. Clin Infect Dis 2010; 50: 49-55.

DOI: $10.1183 / 09031936.00195210$ 